Quì, come altrove, è raffigurato il medico non quello ideale, come alcuni vorrebbero, ma quello vivo, sotto certi aspetti quello di tutti i giorni e di tutti tempi, con le sue virtù e con $i$ suoi difetti, nulla perdendo della sua altissima statura morale.

Da queste brevi considerazioni tratte dai princìpi medico-deontologici di un anonimo della Scuola Salernitana, ancora una volta è interessante constatare che se nei secoli i vari moralisti, teologi, filosofi e tanti altri si cimentarono a trattare l'argomento, impostando il problema sotto punti di vista di volta in volta differenti, essi convergono uniti a quella conclusione che enunciata al tempo di Ippocrate è vera ancora oggi: «Non fare del male, possibilmente giovare».

\title{
De adventu Medici ad aegrotum
}

\section{Anonimi Salernitani}

\section{Testo}

Cum igitur, o medice, ad aegrotum vocaberis, audiutorium sit in nomine Domini. Angelus qui comitatus est Tobiam affectum mentis et egressum corporis comitetur. Intrante tuo a nuntio sciscitare quantum est ex quo infirmus, ad quem vocaris, laboraverit; qualiter ipsum aegritudo invaserit: haec autem sunt necessaria, ut quando ad ipsum accesserit, aegritudinis ejus non omnino inscius videaris; ubi post visa urina, consideratu pulsu, licet per ea aegritudinem non cognoveris, tamen si sinthoma quod praescriveras dixeris, confidet in te, tamquam in autore suae salutis, ad quod summopere laborandum est. Cum igitur ad domum ejus accesseris, antequam ipsum adeas, quaere si conscientiam suam sacerdoti manifestaverit, quod si non fecerit vel faciat vel se facturum promittat; quia si inspecto infirmo consideraris aegritudinis signis super his sermo fit, de sua incipiet desperare salute, quia et te desperare putabit. Ingrediens ad infirmum nec superbientis vultum, nec cupidi praetendas affectum, assurgentes tibi pariter et salutantes humili vultu resalutans et gestu eius sedentibus sedeas. Cum vero jam potus resumpseris, quibusdam verbis interpositis, quibus debes situm regionis illius laudare, dispositionem domus in qua es, si expedit, commendare, vel liberalitatem gentis extollere.

Tandem ad infirmum conversus qualiter se habeat quaeras, et brachium tibi exhiberi praecipias. Et quia ex carne spiritus, in te moti sunt, et infirmus, quia in adventu tuo multum delectatur, vel quia tamquam avarus 
de munere cogitat, propter diversas complexiones tum tui tum infirmi multoties in pulsuum cognitione deciperis. Data ergo securitate aegro interea jam spiritu quiescente pulsum consideres, et attende ne super latus illud jaceat, ne digitos habeat extensos, vel in palmam reductos, et tu cum sinistra sustentes brachium, et usque ad centesimam percussionem ad minus consideres, ubi et diversa pulsuum genera investiges, et astantes ex longa expectatione, verba tua gratiora suscipiant...

Post jubeas tibi afferri urinam, ut aeger et aegritudinem non solum per pulsum sed per urinam cognovisse putet. In urina autem diu attendas colorem, substantiam, quantitatem et contentum; post aegroto cum Dei auxilio salutem promittas. Cum autem ab eo recesseris domesticis ejus dicas ipsum multum laborare, quia ab hoc, si liberabitur, majoris meriti eris et laudis, si vero moriatur testabuntur et a principio de ejus desperasse salute. Unde praeterea moneo ne uxorem vel filiam, vel ancillam oculo cupido respicias; haec Medici excoecant animum operantis, et Dei immutant sententiam cooperantis, et Medicum aegro faciunt honerosum et de se minus bene sperantem. Sis ergo sermone blandus, vitae spectabilis, divino attentius expetens auxilio adjuvari. Cum autem te ad prandium, ut solet fieri, qui domui praesunt invitaverint, nec te importunum ingeras, nec in mensa primus eligas locum, licet Sacerdoti et Medico, ut solet fieri, primus accubitus praeparatur; potum vel cibum non contemnas, nec fastidias quae forte de rure et ergo rusticano pane miliaceo ventris exuriem vix consueveras refrenare. Dum autem comedis per aliquem astantium aegri saepe statum requiras; sic enim de te plurimum confidet infirmus, quem viderit inter delicias sui oblivisci non posse. Surgens autem de coena dicas tibi optime ministratum fuisse, de quo aeger valde laetabitur.

Si vero tempus aegrum cibandi fuerit, opportuno tempore preelecto, cibabis, in iterpolatis quando sunt in vera quiete; in continuis quando est tempus falsae quietis; et in interpolatis tantum ante accessum non tempore ipsius accessi, cibus penitus sit digestus ne natura duplici bello laboret. Si vero accessio anticipat, ut eum cibare non possis, expecta donec accessio transeat, nec tamen statim, quia membra ex praecedente bello defatigata nullum onus a cibis sibi volunt imponi: expecta ergo spatium unius vel duarum horarum. Cibabis autem secundum genus aegritudinis, secundum tempora anni, secundum aetates, variata qualitate et quantitate cibi. Nam ampliori cibabis laborantem interpolata quam continua, et frigidiori in continua quam interpolatis, ampliori hieme et vere, minori aestate et autumno; secundum aetatem, quia pueros sapius reficies quam ju- 
venes, senes pauciori cibo. Sunt etiam cibandi secundum consietudinem, quia si ampliori et grossiori uti cibo consuevit non adeo subtilibus cibis cibandus. Attende etiam ventris constipationem vel fluxum vel medium inter haec; quia si fluxus fit a grossiori incipies cibo, si constipatio est a sorbilibus, dabis pruna et aquam decoctam eorum. Si vero inter haec medium fuerit a liquidioribus tum incipies: dabis ergo primo pruna cocta in aqua, vel granata, vel lac amygdalarum, de quo vero paratum erit exsufflanda est unctuositas quae est in superficie, ne sit fomentum caliditatis quod idem debet fieri de jure gallinae et de aqua in qua resolvitur mica panis; postea da far hordei, et ptisanam dabis in potu, vel aquam ubi positus sit panis, vel aquam bullitam et infrigidatam; et nota quod cibo existente in stomacho nec aquam diureticam nec syrupum dabis, quia haec cogunt cibum indigestum exire vel digestionem retardant.

Debet autem Medicus a principio morbo cum digestivis obviare, cum nec operatio debeat mutari cujus est minister, considerata ergo aegritudinis causa variantur digestiva humorum. Nam si cholera vel alius humor calidus et siccus fuerit in causa da syrupum acetosum, vel syrupum rosarum vel violarum; si melancolia naturalis, vel cholera vitellina maxime in hieme; da oxymel nisi forte natura faciat febrem continuam et tempus sit caloris; haec etiam dentur donec signa digestionis appareant. Item in singulis diebus medicationis signa digestionis attendere et opera debes, quia si in eis major melancoliae copia creverit, diutius eis insistebis; si vero venter fluat lenibus et sorbilibus insiste, ut aquae decoctae violarum, prunorum, et malae: ratio enim mutanda est cum materia varia regnat. Aquam diureticam contra calidam materiam da tempore calido et aetate calida, de semine citreoli, melonum, cucurbitae; sed in frigido tempore et aetati frigidiori contra materiam frigidam da aquam decoctam seminum apii, petroselini, foeniculi, sparagi, brusci, vel radices eorum si vero in aestate de frigida materia laboraverint, vel in hieme de calida, tam ex calidis quam frigidis aqua diuretica praeparetur. Idem si videris statum esse remotum nullo modo purgare praesumas.

Item in flebotomia attende tempus morbi, tempus anni, vires et aetatem aegroti; et a principio autumni usque ad principium veris a sinistro brachio detrahe sanguinem. A principio autem veris a dextro; et propter passionem cerebri venam incide cephalicam, pro morbo spiritualium medianam, pro aegritudine nutrimentorum epaticam, et cum aegro flebotomaveris omnibus curis securitatem inducas. Sanguinis colorem attendas ut si fieri potest tandiu detrahatur sanguis donec color malus mutetur in bonum, et (si) de 
cephalica vena inciditur, caput facies collum fricentur et premantur pectus spatulae et ut tus fiat praecipe; si vero epatica, manibus fricentur et ejus partes in qua fuerit flebotomia etiam aliquantum subleventur, dum vero sanguis exit e naribus appone aquam rosarum vel violarum vel mirtum secundum quod tempus anni exigerit, vel aqua frigida rosa faciem; etiam sanguine sufficienter detracto et ligato brachio, manum tuam aqua frigida infusam superpone brachio ubi non est ligatum usque ad humerum, hoc enim venientem repellit sanguinem et illius partis tumorem prohibet. Hoc facto mulsam syrupi violarum vel rosarum da cum aqua frigida nisi aegritudo sit renovata; quod si fuerit da penidias distemperatas in aqua hordei; si vero non fuerit dabis etiam lac amygdalarum vel micam panis resolutam in aqua. Debet etiam aer fieri obscurus et infrigidari si naturaliter est calidus.

Datis ergo digestivis attende in diebus judicationis quid et unde natura operetur, et si ante diem criticum nulla digestionis signa videris, scies in die critico vel non futuram crisin, vel si fiat fiet in symptomatibus, unde nisi praecedente opere naturae non danda est medicina, sed nec etiam si tota materia expulsa est vi naturae, cum perfecta expulsio cognoscitur per urinam tenuem, per pulsus inanitionem, et per bonum appetitum, et per nocturnam quietem.

Sed quia quidam aegri avaritiae inaebriati veneno, dum vident sine medici auxilio naturam triumphasse de morbo, meritum medici detrahunt pariter et retardant dicentes; quid fecit medicus? natura; iniqua opinione! Syrupis, unctionibus, fomentis videamus salutem inducere quam debit natura, et in alterius intremus labores dicentes morbum post facturum graviorem insultum nisi ei per medicinam succurratur, et sic quod natura fecit imputabitur medico. Si vero facta sit purgatio vi naturae, sed non perfecta, juva naturam purgando unde natura coepit.

Sunt autem diversa signa crisis futurae secundum positionem materiei. Non si fuerit in ore stomachi mordicatio, stomachi abominatio, pruritus labiorum, citatio labii inferioris, tinnitus aurium, dolor major in fronte, crisim futuram per vomitum significat. Si vero dolor est intestinorum inflatio, ventris et laterum gravitas maxime ab umbilico, inferius per secessum crisim expectabis. Si vero lumborum dolor angeatur aegritudine medicina exeunte per urinam fiet crisis, maxime si protensus est tumor et gravitas. Si vero in ano nimia mordicatio et punctura crisis intensius fit per haemorrhoidas, maxime si eas consuevit habere; in muliere vero per menstruum, si gravitas est lumborum et femorum et coxarum, et si tempus menstrui 
aderit. Dolor vero frontis, venarum temporum extensio, perturbatio visus, pruritus narium, fluxus sanguinis e naribus oritur. Superficiei corporis mordicatio, saltus et quaedam jactatio, et in superficie major calor, sudorem promittunt. Et de his omnibus certiores erimus adveniente die critico indicato. Si in praecedenti indicato talia emersere signa, vomitus cum futurus, dato syrupo acetoso, vel sola aqua calida vel frigida, digitis vel penna ori intermissa, juva naturam. Si vero per secessum, moveatur venter cum oleo violarum, cathaplasmetur cum malvis et semine lini, foenugraeci. Si per urinam, da diureticum. Si per haemorroidas, provoca eas; similiter si per menstrua vel per fluxum sanguinis e naribus. Et idem facies si per sudorem; sed si de facili consuevit sudare non est multum cooperiendus. Et nota quia in vere saepius expectare debes crisin futuram per fluxum sanguinis, unde tunc maxime succurrendum est cum phlebotomo; in aestate per sudorem vel vomitum; in autumno et hieme per urinam, vel secessum. Item nota quod in aestate facienda est computatio saepius per tertianarium, maxime si fortis sit aegrotus et materia pauca. In hieme autem per quaternarium et maxime si aeger sit debilis et materia multa; et in aestate potius purganda est per decoctum, in hienem per siccam materiem.

Postquam ad convalescentiam devenerit paullatim ingrossanda est dieta, et mutandus est aer, maxime in aestate, ubi sit in superiori et puriori; et si aer sit corruptus vel ex paludibus, vel ex stagnis, accendatur ignis in domo ubi occupetur. Detrahendus est quandoque sanguis in convalescentia. Si non convaluerit de facili propter malorum humorum reliquias, balneandi sunt etiam et ungendi oleo rosaceo et violaceo, et post balneum dandus ei est syrupus rosarum vel violarum, vel zucara rosata cum frigida, et aquam rosarum odorent et spargatur super faciem et in volis manuum et super loca pulsuum.

Debent etiam tam in aegritudine quam in convalescentia praesentes esse coaequi et de consuetis loqui et consuetos ludos coram eo exercere. Et interim medicus absit; sed tandem ingrediatur hilari vultu dicens: Ecce quanta dixisti, qualiter lusistis, a modo de nobis non curatis, cito demittemus vos cum gaudio et prosperitate. Et sic hoc modo melius se habuerit paulatim facite eos reluitu. Et tandem de petenda licentia consulo ut ejus qui familiarior est aegro a principio favorem acquiras, et ei te et tua omnino exponas, quod quantum sit utile videbis. Impretata ergo licentia quanto poteris diligentius et circumspectius, et quanto poteris honestis promissionibus vade in pace Christo Duce. 


\section{Traduzione}

Quando o Medico serai chiamato presso un malato, il consulto avvenga in nome del Signore. L'Angelo che accompagnò Tobia malato di mente e di corpo ti accompagni. Nell'entrare, come prima cosa informati sulle sofferenze dell'infermo e come sia stato colpito dal male. Queste sono cose necessarie perchè quando sarai alla presenza dell'ammalto non devi sembrare del tutto inconsapevole della sua infermità. Quindi, avendo vista l'urina e considerato il polso é giustificato che tu non abbia ancora conosciuto il male. Quando sarai entrato nella casa dell'infermo, prima che tu sia in sua presenza domanda se si è confessato e che se non l'ha fatto lo faccia o prometta di farlo. Poi se visitato l'ammalto avrai considrato i caratteri della malattia parla di questi e non disperare della guarigione, perché l'ammalto deve credere che guarirà. Entrando dove si trova l'ammalto non apparire superbo e saluta coloro che sono presenti e ti salutano, ed al segno dell'ammalato siediti. Avendo tu accettato quello che ti offrono da bere, tra una parola e l'altra devi lodare quel posto ed elogiare la disposizione della casa e la gentilezza della famiglia.

Finalmente rivolto all'infermo domanda come egli si senta e chiedi che ti porga il braccio. E poiché il tuo animo è turbato dal contatto della carne e poiché l'infermo o è molto compiaciuto del tuo arrivo o pensa avaramente alla retribuzione da darti, questo complesso di circostanze ti potranno ingannare sulla reale valutazione della pulsazioni. Perciò data la tranquillità all'infermo e avendo rasserenato il tuo animo, tasta il polso e guarda bene se l'ammalato giaccia su un fianco o se abbia le dita distese o chiuse a pugno, e tu sostieni il suo braccio con la mano sinistra e senti le pulsazioni almeno fino alla centesima, in modo che tu possa valutare ogni genere di pulsazione. E gli astanti per la tua attenta visita ascolteranno con simpatia le tue parole.

Poi ordina che ti portino l'urina perché tu devi conoscere l'infermità non soltanto con il polso ma anche con l'urina. Nell'urina osserva a lungo il colore, l'odore, la qualità, il contenuto; poi prometti all'ammalto la guarigione con l'aiuto di Dio. Quando ti sarai allontanato dall'ammalto, ai parenti dirai che le condizioni sono gravi e perciò se guarirà tu ne avrai merito, se invece morirà, da te sarà stato detto fin dal principio. Poi ti esorto a non guardare con troppa cupidigia né la moglie, né la figlia, né la domestica dell'infermo. Queste cose distraggono l'animo del medico dalla sua opera e lo rendono malvisto all'infermo e lo fanno apprezzare molto poco. Quando i parenti dell'ammalato poi ti inviteranno a pranzo, come di solito avviene, non essere invadente e non sceglierti il posto; è costume che al Medico e al Sacerdote venga assegnato il primo posto. Non disprezzare le bevande ed il cibo anche se non ti aggrada molto, e mangia il pane anche se fatto di grano turco. E mentre mangi domanda notizie dell'ammalato. In questo modo l'infermo confiderà in te sempre di più vedendo che anche nei divertimenti non viene dimenticato. Poi levandoti da tavola dirai che sei stato trattato molto bene e di ciò l'ammalato sarà molto lieto. 
Quando sarà giunta l'ora di far mangiare l'ammalato, lo farai prima dell'accesso e non durante questo. Infine il cibo sia leggero affinché non vi sia una digestione laboriosa. Se l'accesso anticipa, cosicché tu non possa cibarlo regolarmente, aspetta finché esso passi e non subito dopo, perché le membra stanche per la precedente fatica non devono essere sopraffaticate con cibo. Aspetta perciò che sia passata qualche ora. Gli darai il cibo secondo il tipo della malattia, secondo la stagione, secondo l'età, con variata qualità e quantità. Infatti con più abbondanza farai nutrire chi lavora; durante l'estate a maggior intervallo, mentre nell'inverno più di continuo; più in abbondanza in inverno e in primavera e meno in estate e in autunno; a seconda l'età, perché curerai più spesso con cibo i bambini che i giovani, i vecchi con minor cibo. Si dia il vitto secondo la consuetudine (dell'ammalato), poiché se é solito mangiare abbondantemente non bisogna ridurre troppo il cibo. Osserva la costipazione dell'intestino, o la diarrea, o qualcosa di mezzo tra queste due. Poiché se la costipazione è dovuta a cibo grossolano o a bevande, darai prugne o decotto di esse. Se poi sarà qualcosa di intermedio, incomincerai dai liquidi: darai per primo prugne cotte in acqua, o melograno, o latte di mandorle, da cui bisognerà asportare il grasso che si trova in superficie affinché esso non sia riscaldante, il che bisogna fare pure con il brodo di gallina e la mollica di pane sciolta in acqua. Poi dà pane di orzo e come bevanda una tisana o acqua bollita e poi raffreddata; e bada, quando il cibo è nello stomaco, di non dare acqua diuretica o qualche altro sciroppo poiché queste cose costringono il cibo non ancora digerito ad uscire o ritardano la digestione.

Inoltre il Medico fin dal principio della malattia deve nutrire con sostanze digestive, non dovendo tralasciare questo genere di cura di cui egli è ministro. Considerata dunque la natura della malattia si varino i succhi digestivi; infatti se sarà in causa il colera o qualche altro umore caldo e secco dai sciroppo acetoso, o sciroppo di rose o di viole; se si tratta di «melencolia naturalis» o colera vitellino, specie se d'inverno, dai aceto con miele a meno che non ci sia febbre continua o il tempo sia caldo; queste cose si diano finché non appaiano i segni della digestione. Così durante $\mathrm{i}$ giorni di cura devi osservare $\mathrm{i}$ segni della digestione e gli effetti, poiché se in questi crescerà una maggiore quantità di melancolia, tu più a lungo insisterai su questi; se poi il ventre è meteorico insisti con tenui bevande, come acqua di decotto di viole, di prugne e di mela : infatti il criterio deve mutare a seconda della materia che regna. Dai acqua diuretica contro la materia calda durante l'estate, e nella gioventù dà semi di citriolo, di meloni e di cucurbita; ma in inverno e nella vecchiaia contro la fredda materia dai decotto di semi di apio, di fagiolini, di asparagi, di brusco, ovvero radici di questi se in estate abbiano sofferto per la fredda materia o in inverno per la calda; tanto per la fredda che per la calda si prepari acqua diuretica. Se poi tu vedrai che l'acme è molto lontano, non purgarlo affatto.

Parimenti nella flebotomia osserva il momento della malattia, la stagione dell'anno, le forze e l'età del paziente; e dal principio dell'autunno fino all'inizio 
della primavera estrarrai il sangue dal braccio sinistro. Dall'inizio della primavera in poi dal destro; e per la sofferenza del cervello incidi la vena cefalica, per le malattie dello spirito incidi la mediana, per le malattie della nutrizione la vena basilica, e dopo eseguito il salasso con ogni cura, fa coraggio all'ammalto. Del sangue osserva il colore, e salassa tanto finché il colore del sangue da cattivo diventibuono; e se si incide la vena cefalica si massaggino la faccia, il capo e il collo, e si comprimano il petto e le spalle, specie per provocare la tosse; se si è incisa la basilica, si massaggino con le mani e si sollevino alquanto quelle parti nelle quali sarà stata fatta la flebotomia. Invero quando il sangue esce dalle narici apponi acqua di rose, o di viole, o di mirto a seconda quello che la stagione dell'anno esigerà, ovvero acqua fredda di rose sulla faccia; inoltre sottratto abbastanza sangue e legato il braccio, la tua mano bagnata con acqua fredda metti sul braccio in quella parte dove non è legato fino alla spalla, questo infatti respinge il sangue che viene, impedendo il tumore di quella parte. Fatto questo, dà vino mescolato con miele, sciroppo di viole o di rose mescolato con acqua fredda; a meno che la malattia non sia ricominciata, ché se così sarà dà acqua mescolata con zucchero in acqua di orzo, altrimenti darai il latte di mandorle o moliche di pane sciolte in acqua. L'aria deve essere fresca se per caso facesse caldo.

Dati dunque i digestivi, osserva nei giorni della indicazione in che maniera la natura lavora, e se prima del giorno critico non avrai visto nessun segno di indigestione, cercalo nel giorno critico o nelle crisi future, o nei sintomi; a meno che non si debba dare una medicina a causa della precedente opera della natura, ma se nemmeno tutta la materia è stata espulsa dalle forze della natura, allora la completa espulsione si conosce attraverso il tenue colore dell'urina, o dal polso, o dal buno appetito, o per il riposo notturno. Ma poiché alcuni ammalati, inebriati dal veleno dell'avarizia, quando vedono che senza l'aiuto del medico la natura trionfa sul male, tolgono il merito al medico dicendo: Che cosa ha fatto il medico? Tutto è opera della natura; iniqua opinione! Con sciroppi, unguenti e fomenti noi vediamo ritornare quella salute che la natura diede e ritorniamo ad altro lavoro dicendo che in seguito la malattia sarebbe divenuta più grave senza il soccorso delle medicine, cosi ciò che la natura ha fatto sarà imputato al medico. Se invero sarà stata fatta la purgazione dalle forze della natura, ma non perfettamente, giova soccorrere la natura da dove essa ha cominciato.

Vi sono inoltre diversi segni della crisi futura secondo la posizione della materia. Infatti se ci sarà alla bocca dello stomaco una contrazione dolorosa, uno sgradevole senso allo stomaco, un prurito al labbro inferiore, un tintinnio alle orecchie, un dolore maggiore alla fronte, significa che la crisi avverrà col vomito. Se il dolore è a carico dell'intestino, e vi è meteorismo del ventre, e pesantezza specialmente intorno all'ombellico, apetterai la crisi per la via più bassa. Se invece il dolore è nella regione lombare la crisi avverrà attraverso l'urina, specie se il tumore e la gravità sono molto accentuati. Se nell'ano vi sarà un senso di pun- 
tura troppo forte e di prurito doloroso la crisi avverrà molto più intensa attraverso le emorroidi, specie se quelle datano da molto tempo; nella donna invero attraverso il mestruo, se l'infermità è nella regione lombare, crurale e femorale, e se sarà prossima la mestruazione. Il dolore della fronte e la perturbazione della vista, il prurito delle nerici indicano che il sangue fluirà dal naso. Un senso di prurito doloroso alla superficie del corpo, un'agitazione e un senso di gran calore promettono sudorazione. E di tutti questi segni saremo più certi nell'avvenire del giorno critico. Sei nei segni precedentemente indicati emersero tali sintomi: il vomito con tutto il seguito aiuta con le dita o con una penna introdotta in bocca, dopo aver dato sciroppo acetoso, ovvero sola acqua calda o fredda. Se per la via dell'intestino, si muova il ventre con olio di viole, si facciano cataplasmi con malva e con semi di lino e di fieno greco. Se attraverso l'urina dà diuretici. Se attraverso le emorroidi, provocale; similmente se attraverso i mestrui o per il flusso di sangue dalle narici. E lo stesso farai se attraverso il sudore; ma se quello suda per abitudine non bisogna coprirlo molto. E bada perché in primavera devi aspettare la crisi più spesso attraverso il flusso di sangue, per cui allora specialmente bisogna soccorrere con una flebotomia; in estate attraverso il sudore od il vomito; in autunno e in inverno attraverso l'urina e le deiezioni. Parimenti bada che in estate bisogna fare il calcolo ogni tre giorni specie se l'ammalato è forte e la materia è poca. Nell'inverno invece ogni quattro giorni specie se l'ammalato è debole e la materia è molta; in estate è meglio purgarlo con decotti; in inverno con materie secche.

Man mano che si avvierà alla convalecenza bisogna aumentare gradatamente la dieta, bisogna fargli cambiare aria, specie se in estate, in luoghi più alti e più puri; se l'aria è malsana per paludi o stagni, si arda un fuoco nella stanza dell'ammalato. Di tanto in tanto bisogna togliere del sangue, durante la convalescenza. Se non si riprenderà presto a causa di cattivi umori residui, bisogna fare bagni e ungerlo con olio rosato e di viole e dopo il bagno bisogna dargli sciroppo di rose e di viole; e gli si faccia odorare acqua di rose e si aspergano le palme delle mani e la faccia e i polsi.

Sia durante la malattia che nella convalescenza i parenti non devono mutare abitudini di vita e fare in presenza dell'ammalato i consueti discorsi e giuochi. $\mathrm{E}$ ciò fino a che il medico sarà assente; ma quando questi tornerà a visitare l'infermo dicendo con volto ilare, come per gioco, «ecco quel che dicevate, che non eravate curato bene da me; subito vi licenziamo con gioia e prosperità». $\mathrm{E}$ in questa maniera a poco a poco si licenzierà da essi. Col chiedere licenza in questo modo acquisterai i favori dei familiari e dell'ammalato, parlagli di te e delle tue cose per quel che giudicherai necessario. Chiesta licenza con circospezione e con oneste promesse vai in pace: Christo Duce. 